DOI: https://doi.org/10.46296/ig.v4i8edespdic.0041

\title{
SGC BASADO EN LA ISO 9001:2015, QUE OPTIMICE EL FUNCIONAMIENTO DEL LABORATORIO DE OPERACIONES UNITARIAS UTM
}

\section{QMS BASED ON ISO 9001: 2015, WHICH OPTIMIZES THE OPERATION OF THE UTM UNIT OPERATIONS LABORATORY}

\author{
Saltos-Meza José Gabriel ${ }^{1}$; Burgos-Briones Alfonso ${ }^{2}$ \\ ${ }^{1}$ Universidad Técnica de Manabí, Maestrante del Instituto de Posgrado de la Universidad \\ Técnica de Manabí, UTM. Portoviejo, Ecuador. Correo: jsaltos3973@utm.edu.ec. \\ 2 Universidad Técnica de Manabí, Docente del Instituto de Posgrado de la Universidad Técnica \\ de Manabí, UTM. Portoviejo, Ecuador. Correo: alfonso.burgos@utm.edu.ec.
}

\begin{abstract}
Resumen
La Gestión y control de calidad en los Laboratorios forman parte del diagnóstico principal que garantiza la confiabilidad del resultado procesado dentro del mismo. El objetivo general de esta investigación es el de Diseñar un sistema de gestión de la calidad que optimice el funcionamiento del Laboratorio de Operaciones Unitarias de la Universidad Técnica de Manabí Mediante la aplicación de la normativa ISO 9001:2015. El nivel de investigación empleado fue exploratorio ya que se buscaba conocer la realidad del laboratorio en cuanto al sistema de gestión. El tipo de investigación fue de campo debido a que todos los datos, salvo el soporte intelectual, fueron recabados directamente. Para diagnosticar la situación primeramente se procedió a la observación directa contrastándola contra lo exigido en la norma ISO 9001:2015, posteriormente se aplicó un cuestionario para determinar el nivel de conocimientos de sus integrantes acerca de los SGC. Existen métodos que lleven a una optimización de los recursos y a una mejora continua de la calidad de los servicios en el laboratorio, determinando los diferentes modelos de gestión que se ven afectados por la mala organización. Entre ellos se encuentra el manejo adecuado de los recursos para la mejor ejecución al momento de procesar las actividades. La responsabilidad de la decisión es tomada por el equipo Técnico. Su ejecución implica una organización y un impulso hacia una nueva forma de trabajo enfocada en la estructura de los procesos. La mejora continua y la autoevaluación avanzan sistemáticamente, de esta manera se considera la importancia de una buena gerencia y gestión dentro del laboratorio. Por esta razón es necesario y de gran beneficio enfocar de cerca una gestión y control de calidad aplicado por el laboratorio, con el propósito de relacionar la capacidad técnica empleada en cada fase y así asegurar la eficacia del resultado final.
\end{abstract}

Palabras clave: Calidad, gestión de la calidad, sistemas de gestión de la calidad, ISO 9001:2015.

\footnotetext{
Abstract

The Management and quality control in the Laboratories are part of the main diagnosis that guarantees the reliability of the result processed within it. The general objective of this research is to Design a quality management system that optimizes the operation of the Unit Operations Laboratory of the Technical University of Manabí by applying the ISO 9001: 2015 standard. The level of research used was exploratory since it sought to know the reality of the laboratory in terms of the management system. The type of research was in the field because all the data, except for the intellectual support, were collected directly. To diagnose the situation, firstly, direct observation was made, contrasting it against what is required in the ISO 9001: 2015 standard, later a questionnaire was applied to determine the level of knowledge of its members about QMS. There are methods that lead to an optimization of resources and a continuous improvement of Información del manuscrito:

Fecha de recepción: 29 de octubre de 2021.

Fecha de aceptación: 07 de diciembre de 2021.

Fecha de publicación: 08 de diciembre de 2021.
} 
the quality of services in the laboratory, determining the different management models that are affected by poor organization. Among them is the adequate management of resources for the best execution when processing activities. The responsibility for the decision is taken by the Technical team. Its execution implies an organization and an impulse towards a new way of working focused on the structure of the processes. Continuous improvement and self-evaluation progress systematically, in this way the importance of good management and management within the laboratory is considered. For this reason, it is necessary and of great benefit to closely focus on management and quality control applied by the laboratory, in order to relate the technical capacity used in each phase and thus ensure the effectiveness of the final result.

Keywords: Quality, quality management, quality management systems, ISO 9001: 2015.

\section{Introducción}

El presente estudio muestra el diseño de un Sistema de Gestión de Calidad en el Laboratorio de Operaciones Unitarias de la Universidad Técnica de Manabí, este sistema de gestión garantizará el mejor funcionamiento del laboratorio, facilitará también el desarrollo de las actividades y un mayor acercamiento al principio de la calidad interna, siguiendo la normativa ISO 9001:2015.

Según los autores Tomás J. Fontalvo y Efraín J. De La Hoz en su artículo científico publicado en la revista SciELO en el año 2018 titulado: Diseño e Implementación de un Sistema de Gestión de la Calidad ISO 9001:2015 en una Universidad Colombiana, menciona que estos sistemas aportan al sector universitario y a la sociedad en general las estructuras operativas.

Por otra parte, este estudio pretende convertirse en un antecedente más para aquellas investigaciones que se encuentren vinculadas directa 0 indirectamente con el mismo; por lo que sirve de herramienta de apoyo para otros estudiantes que se encuentren interesados en sistemas de gestión de calidad de procesos, basados en la Norma ISO 9001:2015.

\section{Materiales y métodos}

La presente investigación se rige bajo un diseño de campo, ya que este diseño permite establecer una interacción entre los objetivos y la realidad donde ocurren los hechos, la observación y la recolección de datos se realiza directamente de la realidad en su situación natural permitiendo profundizar en la comprensión de los hallazgos 
encontrados con la aplicación de los instrumentos, ofreciendo una percepción de la realidad objeto de estudio más rica en cuanto al conocimiento de la misma. Los datos obtenidos a partir de la investigación bajo un diseño de campo son denominados datos primarios.

La población sobre la cual se basa esta investigación está conformada por el Laboratorio de Operaciones Unitarias de la Universidad Técnica de Manabí conforman el proceso medular de la investigación, el cual se describe a continuación; 1500 estudiantes de las carreras de ingeniería industrial y de ingeniería química que realizan prácticas en el Laboratorio de Operaciones Unitarias de la Universidad Técnica de Manabí. En lo que refiere a la población de los estudiantes que corresponde a 1500 se aplicó formula de muestra cómo se detalla a continuación.

$\mathrm{N}=$ Población o universo $=1500$

$\mathrm{P}=$ Probabilidad de ocurrencia = 0.50

$\mathrm{Q}=$ Probabilidad de no ocurrencia = 0.50

$Z=$ Nivel de confianza $=1.96$

$\mathrm{e}=$ Nivel de significancia $=0.05$

$\mathrm{n}=$ Muestra: 278

$$
\begin{gathered}
\mathrm{n}=\frac{\mathrm{N} \sigma^{2} \mathrm{Z}^{2}}{\mathrm{e}^{2}(\mathrm{~N}-1)+\sigma^{2} \mathrm{Z}^{2}} \\
\mathrm{n}=\frac{(1500)(0,5)^{2}(1,96)^{2}}{(0,05)^{2}[(1500-1)]+\left[(0,5)^{2}(1,96)^{2}\right]} \\
\mathrm{n}=\frac{(1500(0,25)(3,8416)}{(0,0025)(1499)+(0,25)(3,8416)} \\
\mathrm{n}=\frac{533,022}{1,385+0,9604} \\
n-533,022
\end{gathered}
$$

Tabla1. Población y muestra.

\begin{tabular}{ccc}
\hline Universo & Población & Muestra \\
\hline Estudiantes de & 1500 & 278 \\
las carreras de \\
ingeniería \\
industrial y de \\
ingeniería \\
química \\
Total & & \\
& & \\
\hline
\end{tabular}

Autor: (Saltos 2021)

Delimitación espacial: Portoviejo, Laboratorio de Operaciones Unitarias de la Facultad de Ciencias Matemáticas, Físicas y Químicas de la Universidad Técnica de Manabí.

Para la determinación de conocimientos sobre el sistema de calidad del personal se utilizará un cuestionario de veinte (20) ítems con criterios de selecciones cerradas y dicotómicas donde se hacen preguntas sobre la presencia de algunas de las características más resaltantes de un sistema de gestión de la calidad para los estudiantes, En 
esta encuesta se han anexado preguntas donde la respuestas afirmativas pueden ser rechazadas si el encuestado no conoce el porqué de su respuesta a los fines de evitar recabar informaciones erróneas.

En atención a la problemática, mediante los resultados obtenidos, se hizo necesario efectuar el análisis cualicuantitativo pertinente a los diferentes ítems presentados en el instrumento.

\section{Resultados}

En la presente investigación se realizó una encuesta de manera digital a 278 estudiantes de las carreras de Ingeniería Química e Ingeniería Industrial de la Universidad Técnica de Manabí, con la finalidad de obtener información directa y precisa y así los datos obtenidos no fueran a perjudicar esta investigación.

Realizando una síntesis acerca de las preguntas que formaron parte de esta encuesta lo que se necesitaba conocer era si un Sistema de Gestión de Calidad basado en la norma ISO 9001:2015 ayudaría a la optimización y funcionamiento del laboratorio de operaciones unitarias, junto a esta pregunta que era la principal se cuestionó también a los estudiantes si conocen algunos elementos con los que debía contar el laboratorio ya que serían parte clave para el desarrollo del SGC.

Los resultados que se obtuvieron están dentro de lo imaginado ya que la totalidad de los encuestados respondieron que estaban totalmente de acuerdo con la implementación de un SGC para el Laboratorio de Operaciones Unitarias.

\section{Ítems 1. ¿El laboratorio de Operaciones Unitarias, posee instalaciones adecuadas para el servicio prestado?}

Tabla 2. Instalaciones adecuadas para el servicio prestado.

\begin{tabular}{ccc}
\hline Ítems & Frecuencia & Porcentaje \% \\
\hline $\begin{array}{c}\text { Totalmente de } \\
\text { acuerdo }\end{array}$ & $\mathbf{2 7 3}$ & $\mathbf{9 8}$ \\
$\begin{array}{c}\text { Parcialmente } \\
\text { de acuerdo }\end{array}$ & $\mathbf{0}$ & $\mathbf{0}$ \\
$\begin{array}{c}\text { Ni de acuerdo, } \\
\text { ni en }\end{array}$ & $\mathbf{5}$ & $\mathbf{2}$ \\
$\begin{array}{c}\text { desacuerdo } \\
\text { arcialmente }\end{array}$ & $\mathbf{0}$ & $\mathbf{0}$ \\
$\begin{array}{c}\text { en desacuerdo } \\
\text { Totalmente } \\
\text { desacuerdo } \\
\text { Total }\end{array}$ & $\mathbf{0}$ & $\mathbf{0}$ \\
\hline
\end{tabular}

Fuente: Saltos (2021)

De acuerdo a la opinión de todos los encuestados (98\%), estuvieron totalmente de acuerdo que el laboratorio posee instalaciones 
adecuadas para prestar sus

servicios de prácticas a los

estudiantes.

Ítems 2. ¿El personal que labora en el laboratorio de Operaciones Unitarias, cuenta con el equipamiento necesario para prestar su servicio?

Tabla 3.

\begin{tabular}{lcc}
\hline \multicolumn{1}{c}{ ítEMS } & FRECUENCIA & $\begin{array}{c}\text { PORCENTAJE } \\
\%\end{array}$ \\
\hline $\begin{array}{l}\text { Totalmente } \\
\text { de acuerdo }\end{array}$ & 268 & 96 \\
$\begin{array}{l}\text { Parcialmente } \\
\text { de acuerdo }\end{array}$ & 4 & 2 \\
Ni de & & \\
$\begin{array}{l}\text { acuerdo, ni en } \\
\text { desacuerdo }\end{array}$ & 6 & $\mathbf{2}$ \\
$\begin{array}{l}\text { Parcialmente } \\
\text { en } \\
\text { desacuerdo }\end{array}$ & 0 & $\mathbf{0}$ \\
$\begin{array}{l}\text { Totalmente } \\
\text { desacuerdo }\end{array}$ & 0 & $\mathbf{0}$ \\
\begin{tabular}{l} 
Total \\
\hline
\end{tabular} & $\mathbf{2 7 8}$ & $\mathbf{1 0 0}$
\end{tabular}

Fuente: Saltos (2021)

Interpretación de los resultados:

De todos los encuestados el (96\%), manifestaron que el personal cuenta con el equipamiento necesario para prestar su servicio, proyectando una buena imagen del personal, y dejando una buena impresión a todos los que utilizan el laboratorio.

Ítems 3. ¿El laboratorio de Operaciones Unitarias, cuenta con los equipos y tecnología adecuada para prestar su servicio?
Tabla 4. Cuenta con los equipos y tecnología adecuada para prestar su servicio.

\begin{tabular}{lcc}
\hline ÍTEMS & FRECUENCIA & PORCENTAJE \% \\
\hline $\begin{array}{l}\text { Totalmente } \\
\text { de acuerdo }\end{array}$ & 35 & 13 \\
$\begin{array}{l}\text { Parcialmente } \\
\text { de acuerdo }\end{array}$ & 235 & 84 \\
$\begin{array}{l}\mathrm{Ni} \text { de } \\
\text { acuerdo, ni } \\
\text { en } \\
\text { desacuerdo }\end{array}$ & 8 & 3 \\
$\begin{array}{l}\text { Parcialmente } \\
\text { en } \\
\text { desacuerdo }\end{array}$ & 0 & 0 \\
$\begin{array}{l}\text { Totalmente } \\
\text { desacuerdo }\end{array}$ & 0 & 0 \\
Total & $\mathbf{2 7 8}$ & $\mathbf{1 0 0}$ \\
\hline
\end{tabular}

Fuente: Saltos (2021)

Interpretación de los resultados:

Aun cuando la mayoría (84\%) de los encuestados, estuvieron parcialmente de acuerdo en que el laboratorio, cuenta con los equipos adecuados para prestar su servicio, se debía tener en cuenta cada uno de ellos y estar en constante actualización.

Ítems 4. ¿Considera usted necesario un Sistema de Gestión de Calidad para mejorar la calidad de los servicios?

Tabla 5. Sistema de Gestión de Calidad.

\begin{tabular}{lcc}
\hline ÍTEMS & FRECUENCIA & $\begin{array}{l}\text { PORCENTAJE } \\
\%\end{array}$ \\
\hline $\begin{array}{l}\text { Totalmente } \\
\text { de acuerdo }\end{array}$ & 278 & $\mathbf{1 0 0}$ \\
$\begin{array}{l}\text { Parcialmente } \\
\text { de acuerdo }\end{array}$ & 0 & $\mathbf{0}$ \\
\hline
\end{tabular}




\begin{tabular}{lcc}
$\begin{array}{l}\mathrm{Ni} \\
\text { acuerdo, } \mathrm{ni} \\
\text { en }\end{array}$ & 0 & 0 \\
desacuerdo & & \\
$\begin{array}{l}\text { Parcialmente } \\
\text { en } \\
\text { desacuerdo }\end{array}$ & 0 & 0 \\
$\begin{array}{l}\text { Totalmente } \\
\text { desacuerdo }\end{array}$ & 0 & 0 \\
Total & $\mathbf{2 7 8}$ & $\mathbf{1 0 0}$ \\
\hline
\end{tabular}

Fuente: Saltos (2021)

Interpretación de los resultados:

El total (100\%) de los encuestados manifestaron que estaban totalmente de acuerdo en la necesidad de un Sistema de Gestión de Calidad para mejorar la calidad del servicio y funcionamiento del laboratorio.

Ítems 5. ¿En el laboratorio de Operaciones Unitarias se necesita poseer inmuebles acordes a un Sistema de Gestión de Calidad?

Tabla 6. Sistema de Gestión de Calidad.

\begin{tabular}{|c|c|c|}
\hline ÍTEMS & FRECUENCIA & $\begin{array}{l}\text { PORCENTAJE } \\
\%\end{array}$ \\
\hline $\begin{array}{l}\text { Totalmente de } \\
\text { acuerdo }\end{array}$ & 278 & 100 \\
\hline $\begin{array}{l}\text { Parcialmente } \\
\text { de acuerdo }\end{array}$ & 0 & 0 \\
\hline $\begin{array}{l}\mathrm{Ni} \text { de acuerdo, } \\
\text { ni en } \\
\text { desacuerdo }\end{array}$ & 0 & 0 \\
\hline $\begin{array}{l}\text { Parcialmente } \\
\text { en desacuerdo }\end{array}$ & 0 & 0 \\
\hline $\begin{array}{l}\text { Totalmente } \\
\text { desacuerdo }\end{array}$ & 0 & 0 \\
\hline Total & 278 & 100 \\
\hline
\end{tabular}

Fuente: Saltos (2021)
Interpretación de los resultados:

El total (100\%) de los encuestados, estuvieron totalmente de acuerdo en que la empresa cuente con inmuebles acordes a las exigencias de un Sistema de Gestión de Calidad, permitiendo al laboratorio desarrollar sus actividades e incrementar su capacidad, conjuntamente con el mantenimiento de los mismos.

Ítems 6. ¿En el laboratorio de Operaciones Unitarias se cuenta con los insumos requeridos por un Sistema de Gestión de Calidad?

Tabla 7. Insumos requeridos por un Sistema de Gestión de Calidad.

\begin{tabular}{|c|c|c|}
\hline ÍTEMS & FRECUENCIA & PORCENTAJE \% \\
\hline $\begin{array}{l}\text { Totalmente } \\
\text { de acuerdo }\end{array}$ & 278 & 100 \\
\hline $\begin{array}{l}\text { Parcialmente } \\
\text { de acuerdo }\end{array}$ & 0 & 0 \\
\hline $\begin{array}{lc}\mathrm{Ni} & \text { de } \\
\text { acuerdo, } & \text { ni } \\
\text { en } & \\
\text { desacuerdo }\end{array}$ & 0 & 0 \\
\hline $\begin{array}{l}\text { Parcialmente } \\
\text { en } \\
\text { desacuerdo }\end{array}$ & 0 & 0 \\
\hline $\begin{array}{l}\text { Totalmente } \\
\text { desacuerdo }\end{array}$ & 0 & 0 \\
\hline Total & 278 & 100 \\
\hline
\end{tabular}

Fuente: Saltos (2021)

Interpretación de los resultados:

Todos los encuestados (100\%), estuvieron totalmente de acuerdo en que el laboratorio cuenta con los 
insumos necesarios para la puesta en marcha de un Sistema de Gestión de Calidad.

Ítems 7. ¿En el laboratorio de Operaciones Unitarias, cuenta con los instrumentos y herramientas requeridos por un Sistema de Gestión de Calidad?

Tabla 8. ¿Instrumentos y herramientas requeridos por un Sistema de Gestión de Calidad?

\begin{tabular}{lcc}
\hline ÍTEMS & FRECUENCIA & $\begin{array}{l}\text { PORCENTAJE } \\
\%\end{array}$ \\
\hline $\begin{array}{l}\text { Totalmente de } \\
\text { acuerdo }\end{array}$ & 209 & $\mathbf{7 5}$ \\
$\begin{array}{l}\text { Parcialmente } \\
\text { de acuerdo }\end{array}$ & 69 & $\mathbf{2 5}$ \\
$\begin{array}{l}\text { Ni de acuerdo, } \\
\text { ni } \\
\text { desacuerdo }\end{array}$ & 0 & $\mathbf{0}$ \\
$\begin{array}{l}\text { Parcialmente } \\
\text { en desacuerdo }\end{array}$ & 0 & $\mathbf{0}$ \\
$\begin{array}{l}\text { Totalmente } \\
\text { desacuerdo }\end{array}$ & 0 & $\mathbf{0}$ \\
Total & $\mathbf{2 7 8}$ & $\mathbf{1 0 0}$ \\
\hline
\end{tabular}

Fuente: Saltos (2021)

Interpretación de los resultados:

La gran mayoría $(75 \%)$ de los encuestados, estuvieron totalmente de acuerdo, en que la empresa cuenta con los instrumentos y herramientas requeridos para la puesta en marcha de un Sistema de Gestión de Calidad, siendo requerida la implementación de nuevas tecnologías para automatizar los procedimientos. Sólo una pequeña parte (25\%), estuvo parcialmente de acuerdo.

Ítems 8. ¿El personal con el que se cuenta en el laboratorio de Operaciones Unitarias, cuenta con las habilidades requeridas por un Sistema de Gestión de Calidad?

Tabla 9. Habilidades requeridas.

\begin{tabular}{|c|c|c|}
\hline ÍTEMS & FRECUENCIA & PORCENTAJE \% \\
\hline $\begin{array}{l}\text { Totalmente } \\
\text { de acuerdo }\end{array}$ & 0 & 0 \\
\hline $\begin{array}{l}\text { Parcialmente } \\
\text { de acuerdo }\end{array}$ & 48 & 17 \\
\hline $\begin{array}{ll}\mathrm{Ni} & \mathrm{de} \\
\text { acuerdo, } & \mathrm{ni} \\
\text { en } & \\
\text { desacuerdo }\end{array}$ & 230 & 83 \\
\hline $\begin{array}{l}\text { Parcialmente } \\
\text { en } \\
\text { desacuerdo }\end{array}$ & 0 & 0 \\
\hline $\begin{array}{l}\text { Totalmente } \\
\text { desacuerdo }\end{array}$ & 0 & 0 \\
\hline Total & 278 & 100 \\
\hline
\end{tabular}

Fuente: Saltos (2021)

Interpretación de los resultados:

De los encuestados el $17 \%$ estaba parcialmente de acuerdo, mientras que un $83 \%$ la gran mayoría indicaron estar ni de acuerdo ni en desacuerdo en que el personal que labora cuente con las habilidades requeridas por un Sistema de Gestión de Calidad.

Ítems 9. ¿El personal que labora en el laboratorio de Operaciones Unitarias, cuenta con el conocimiento requerido por un Sistema de Gestión de Calidad? 
Tabla 10. Conocimiento requerido por un Sistema de Gestión de Calidad.

\begin{tabular}{lcc}
\hline ÍTEMS & FRECUENCIA & $\begin{array}{l}\text { PORCENTAJE } \\
\%\end{array}$ \\
\hline $\begin{array}{l}\text { Totalmente de } \\
\text { acuerdo }\end{array}$ & 278 & $\mathbf{1 0 0}$ \\
$\begin{array}{l}\text { Parcialmente } \\
\text { de acuerdo }\end{array}$ & 0 & $\mathbf{0}$ \\
$\begin{array}{l}\text { Ni de acuerdo, } \\
\text { ni en } \\
\text { desacuerdo }\end{array}$ & 0 & $\mathbf{0}$ \\
$\begin{array}{l}\text { Parcialmente } \\
\text { en desacuerdo }\end{array}$ & 0 & $\mathbf{0}$ \\
$\begin{array}{l}\text { Totalmente } \\
\text { desacuerdo }\end{array}$ & 0 & $\mathbf{0}$ \\
Total & 278 & $\mathbf{1 0 0}$ \\
\hline
\end{tabular}

Fuente: Saltos (2021)

Interpretación de los resultados:

Todos los encuestados el $100 \%$ indicaron que están totalmente de acuerdo en que el personal que trabaja en el laboratorio cuenta con los conocimientos requeridos por un Sistema de Gestión de Calidad.

Ítems 10. ¿Considera usted necesaria la inclusión de las responsabilidades de la alta dirección dentro del Sistema de Gestión de Calidad?

Tabla 11. Responsabilidad de la dirección.

\begin{tabular}{lcc}
\hline ÍTEMS & FRECUENCIA & $\begin{array}{l}\text { PORCENTAJE } \\
\%\end{array}$ \\
\hline $\begin{array}{l}\text { Totalmente } \\
\text { de acuerdo }\end{array}$ & 278 & $\mathbf{1 0 0}$ \\
$\begin{array}{l}\text { Parcialmente } \\
\text { de acuerdo }\end{array}$ & 0 & $\mathbf{0}$ \\
$\begin{array}{l}\mathrm{Ni} \text { de } \\
\text { acuerdo, ni en } \\
\text { desacuerdo }\end{array}$ & 0 & $\mathbf{0}$ \\
\hline
\end{tabular}

\begin{tabular}{lcc}
\hline $\begin{array}{l}\text { Parcialmente } \\
\text { en } \\
\text { desacuerdo }\end{array}$ & 0 & $\mathbf{0}$ \\
$\begin{array}{l}\text { Totalmente } \\
\text { desacuerdo }\end{array}$ & 0 & $\mathbf{0}$ \\
Total & 278 & $\mathbf{1 0 0}$ \\
\hline
\end{tabular}

Fuente: Saltos (2021)

Interpretación de los resultados:

Todos los encuestados el $100 \%$ estuvieron totalmente de acuerdo en que es necesaria la inclusión de la responsabilidad de la dirección dentro del Sistema de Gestión de Calidad.

Ítems 11. ¿Considera usted necesaria la inclusión de la gestión de los recursos de seguimiento y control dentro del Sistema de Gestión de Calidad?

Tabla 12. ¿Considera usted necesaria la inclusión de la gestión de los recursos dentro del Sistema de Gestión de Calidad?

\begin{tabular}{lll}
\hline ITEMS & FRECUENCIA & PORCENTAJE \% \\
\hline $\begin{array}{l}\text { Totalmente } \\
\text { de acuerdo }\end{array}$ & 278 & $\mathbf{1 0 0}$ \\
$\begin{array}{l}\text { Parcialmente } \\
\text { de acuerdo }\end{array}$ & 0 & $\mathbf{0}$ \\
$\mathrm{Ni} \quad$ de & 0 & $\mathbf{0}$ \\
$\begin{array}{l}\text { acuerdo, ni } \\
\text { en } \\
\text { desacuerdo }\end{array}$ & \\
$\begin{array}{l}\text { Parcialmente } \\
\text { en }\end{array}$ & 0 & $\mathbf{0}$ \\
desacuerdo & & \\
$\begin{array}{l}\text { Totalmente } \\
\text { desacuerdo }\end{array}$ & 0 & $\mathbf{0}$ \\
Total & $\mathbf{2 7 8}$ & $\mathbf{1 0 0}$
\end{tabular}

Fuente: Saltos (2021) 
Interpretación de los resultados:

Todos los encuestados (100\%), estuvieron totalmente de acuerdo con la inclusión de la gestión de los recursos dentro del Sistema de Gestión de Calidad, a fin de proporcionar los recursos necesarios para implementar y mantener el sistema, y mejorar continuamente su eficacia.

Ítems 12. ¿Considera usted necesaria la inclusión de los requisitos para los productos y servicios dentro del Sistema de Gestión de Calidad?

Tabla 13. Inclusión de la realización del servicio.

\begin{tabular}{lcc}
\hline ITEMS & FRECUENCIA & $\begin{array}{l}\text { PORCENTAJE } \\
\%\end{array}$ \\
\hline $\begin{array}{l}\text { Totalmente } \\
\text { de acuerdo }\end{array}$ & 278 & $\mathbf{1 0 0}$ \\
$\begin{array}{l}\text { Parcialmente } \\
\text { de acuerdo }\end{array}$ & 0 & $\mathbf{0}$ \\
$\begin{array}{l}\mathrm{Ni} \text { de } \\
\text { acuerdo, ni en } \\
\text { desacuerdo }\end{array}$ & 0 & $\mathbf{0}$ \\
$\begin{array}{l}\text { Parcialmente } \\
\text { en } \\
\text { desacuerdo }\end{array}$ & 0 & $\mathbf{0}$ \\
$\begin{array}{l}\text { Totalmente } \\
\text { desacuerdo }\end{array}$ & 0 & $\mathbf{0}$ \\
Total & 278 & $\mathbf{1 0 0}$ \\
\hline
\end{tabular}

Fuente: Saltos (2021)

Interpretación de los resultados:

El total (100\%) de los encuestados, manifestaron que estaban totalmente de acuerdo con la inclusión de la realización del servicio dentro del Sistema de Gestión de Calidad con la finalidad de Planificar y desarrollar los procesos necesarios para la realización del servicio.

Ítems 13. ¿Considera usted necesaria la inclusión del control de los procesos, medición, análisis y mejor del servicio, dentro del Sistema de Gestión de Calidad?

Tabla 14. Inclusión de la medición, análisis y mejor del servicio.

\begin{tabular}{lcc}
\hline İTEMS & FRECUENCIA & PORCENTAJE \% \\
\hline $\begin{array}{l}\text { Totalmente } \\
\text { de acuerdo }\end{array}$ & 278 & 100 \\
$\begin{array}{l}\text { Parcialmente } \\
\text { de acuerdo }\end{array}$ & 0 & 0 \\
$\begin{array}{l}\mathrm{Ni} \quad \text { de } \\
\text { acuerdo, ni } \\
\text { en } \\
\text { desacuerdo }\end{array}$ & 0 & $\mathbf{0}$ \\
$\begin{array}{l}\text { Parcialmente } \\
\text { en } \\
\text { desacuerdo }\end{array}$ & 0 & $\mathbf{0}$ \\
$\begin{array}{l}\text { Totalmente } \\
\text { desacuerdo }\end{array}$ & 0 & $\mathbf{0}$ \\
Total & 278 & $\mathbf{1 0 0}$ \\
\hline
\end{tabular}

Fuente: Saltos (2021)

Interpretación de los resultados:

Todos los encuestados (100\%), manifestaron que estaban totalmente de acuerdo con la inclusión de la medición, análisis y mejora del servicio, a fin de explicar que hay que medir, para obtener la información de cómo mejorar el sistema. 
Ítems 14. ¿Considera usted necesaria la inclusión de la comunicación interna y externa dentro del Sistema de Gestión de Calidad?

Tabla 15. Inclusión de la comunicación facultad - docentes- estudiantes.

\begin{tabular}{lcc}
\hline ÍTEMS & FRECUENCIA & $\begin{array}{l}\text { PORCENTAJE } \\
\%\end{array}$ \\
\hline $\begin{array}{l}\text { Totalmente } \\
\text { de acuerdo }\end{array}$ & 278 & $\mathbf{1 0 0}$ \\
$\begin{array}{l}\text { Parcialmente } \\
\text { de acuerdo }\end{array}$ & 0 & 0 \\
$\begin{array}{l}\text { Ni de } \\
\text { acuerdo, ni en } \\
\text { desacuerdo }\end{array}$ & 0 & 0 \\
$\begin{array}{l}\text { Parcialmente } \\
\text { en } \\
\text { desacuerdo }\end{array}$ & 0 & $\mathbf{0}$ \\
$\begin{array}{l}\text { Totalmente } \\
\text { desacuerdo }\end{array}$ & 0 & $\mathbf{0}$ \\
\begin{tabular}{l} 
Total \\
\hline
\end{tabular} & 278 & $\mathbf{1 0 0}$ \\
\hline
\end{tabular}

Fuente: Saltos (2021)

Interpretación de los resultados:

Todos los encuestados (100\%), estuvieron totalmente de acuerdo con la inclusión de la comunicación facultad - docentes y estudiantes dentro del Sistema de Gestión de Calidad, ya que la mejora de la comunicación interna.

Ítems 15. ¿Considera usted necesaria la inclusión de la infraestructura dentro del Sistema de Gestión de Calidad?
Tabla 16. Inclusión de la infraestructura.

\begin{tabular}{lcc}
\hline ÍTEMS & FRECUENCIA & PORCENTAJE \% \\
\hline $\begin{array}{l}\text { Totalmente } \\
\text { de acuerdo }\end{array}$ & 278 & $\mathbf{1 0 0}$ \\
$\begin{array}{l}\text { Parcialmente } \\
\text { de acuerdo }\end{array}$ & 0 & $\mathbf{0}$ \\
$\begin{array}{l}\mathrm{Ni} \text { de } \\
\text { acuerdo, ni } \\
\text { en } \\
\text { desacuerdo }\end{array}$ & 0 & $\mathbf{0}$ \\
$\begin{array}{l}\text { Parcialmente } \\
\text { en } \\
\text { desacuerdo }\end{array}$ & 0 & $\mathbf{0}$ \\
$\begin{array}{l}\text { Totalmente } \\
\text { desacuerdo }\end{array}$ & 0 & $\mathbf{0}$ \\
Total & 278 & $\mathbf{1 0 0}$ \\
\hline
\end{tabular}

Fuente: Saltos (2021)

Interpretación de los resultados:

El total (100\%) de los encuestados, estuvieron totalmente de acuerdo con la inclusión de la infraestructura dentro del Sistema de Gestión de Calidad, a fin de determinar qué infraestructura requiere el sistema de gestión diseñado, proporcionar la infraestructura que se ha determinado como necesaria y mantener la infraestructura en perfectas condiciones para su uso cuando se precise.

Ítems 16. ¿Considera usted necesaria la inclusión del ambiente para la operación de los procesos dentro del Sistema de Gestión de Calidad? 
Tabla 17. Inclusión del ambiente de trabajo dentro del Sistema de Gestión de Calidad.

\begin{tabular}{lcc}
\hline ÍTEMS & FRECUENCIA & $\begin{array}{l}\text { PORCENTAJE } \\
\%\end{array}$ \\
\hline $\begin{array}{l}\text { Totalmente } \\
\text { de acuerdo }\end{array}$ & 278 & 100 \\
$\begin{array}{l}\text { Parcialmente } \\
\text { de acuerdo }\end{array}$ & 0 & 0 \\
$\begin{array}{l}\mathrm{Ni} \text { de } \\
\text { acuerdo, ni en } \\
\text { desacuerdo }\end{array}$ & 0 & 0 \\
$\begin{array}{l}\text { Parcialmente } \\
\text { en } \\
\text { desacuerdo }\end{array}$ & 0 & $\mathbf{0}$ \\
$\begin{array}{l}\text { Totalmente } \\
\text { desacuerdo }\end{array}$ & 0 & $\mathbf{0}$ \\
Total & $\mathbf{2 7 8}$ & $\mathbf{1 0 0}$ \\
\hline
\end{tabular}

Fuente: Saltos (2021)

Interpretación de los resultados:

El total (100\%) de los encuestados, manifestó estar totalmente de acuerdo con la inclusión del ambiente de trabajo dentro del Sistema de Gestión de Calidad, a fin de determinar la atmósfera profesional en la que destacan aspectos como la atención y el trato al cliente, la organización de los puestos de trabajo, la eficiencia, el grado de colaboración de todas las personas, tanto entre empleados como entre empleados directivos.

Ítems 17 ¿Considera usted necesaria la inclusión de los estándares de calidad dentro del Sistema de Gestión de Calidad?
Tabla 18. Inclusión de los estándares de calidad dentro del Sistema de Gestión de Calidad.

\begin{tabular}{lcc}
\hline ÍTEMS & FRECUENCIA & PORCENTAJE \% \\
\hline $\begin{array}{l}\text { Totalmente } \\
\text { de acuerdo }\end{array}$ & 278 & $\mathbf{1 0 0}$ \\
$\begin{array}{l}\text { Parcialmente } \\
\text { de acuerdo }\end{array}$ & 0 & $\mathbf{0}$ \\
$\begin{array}{l}\mathrm{Ni} \text { de } \\
\text { acuerdo, ni } \\
\text { en } \\
\text { desacuerdo }\end{array}$ & 0 & $\mathbf{0}$ \\
$\begin{array}{l}\text { Parcialmente } \\
\text { en } \\
\text { desacuerdo }\end{array}$ & 0 & $\mathbf{0}$ \\
$\begin{array}{l}\text { Totalmente } \\
\text { desacuerdo }\end{array}$ & 0 & $\mathbf{0}$ \\
Total & 278 & $\mathbf{1 0 0}$ \\
\hline
\end{tabular}

Fuente: Saltos (2021)

Interpretación de los resultados:

Todos los encuestados (100\%), manifestaron que estaban totalmente de acuerdo con la inclusión de los estándares de calidad dentro del Sistema de Gestión de Calidad, los cuales definen un conjunto de criterios que guían la forma en que se aplica el servicio. Si no se sigue ninguna metodología siempre habrá falta de calidad.

Items 18. ¿Considera usted necesaria la inclusión de la visión integral dentro del Sistema de Gestión de Calidad? 
Tabla 19. Inclusión de la visión integral dentro del Sistema de Gestión de Calidad.

\begin{tabular}{lcc}
\hline ÍTEMS & FRECUENCIA & $\begin{array}{l}\text { PORCENTAJE } \\
\%\end{array}$ \\
\hline $\begin{array}{l}\text { Totalmente de } \\
\text { acuerdo }\end{array}$ & 278 & $\mathbf{1 0 0}$ \\
$\begin{array}{l}\text { Parcialmente } \\
\text { de acuerdo }\end{array}$ & 0 & $\mathbf{0}$ \\
$\begin{array}{l}\text { Ni de acuerdo, } \\
\text { ni en } \\
\text { desacuerdo }\end{array}$ & 0 & 0 \\
$\begin{array}{l}\text { Parcialmente } \\
\text { en desacuerdo }\end{array}$ & 0 & $\mathbf{0}$ \\
$\begin{array}{l}\text { Totalmente } \\
\text { desacuerdo }\end{array}$ & 0 & $\mathbf{0}$ \\
Total & $\mathbf{2 7 8}$ & $\mathbf{1 0 0}$ \\
\hline
\end{tabular}

Fuente: Saltos (2021)

Interpretación de los resultados:

Todos los encuestados (100\%), estuvieron totalmente de acuerdo con la inclusión de la visión integral dentro del Sistema de Gestión de Calidad, a fin de adaptarse a los constantes cambios que enfrentan actualmente las organizaciones, mejorando el nivel de eficiencia y de calidad de los servicios, con el apoyo de herramientas de mejoramiento continuo de la calidad y productividad.

Ítems 19. ¿Considera usted necesaria la inclusión de la optimización de los procesos dentro del Sistema de Gestión de Calidad?
Tabla 20. Inclusión de la optimización de los procesos dentro del Sistema de Gestión de Calidad.

\begin{tabular}{|c|c|c|}
\hline ÍTEMS & FRECUENCIA & PORCENTAJE \% \\
\hline $\begin{array}{l}\text { Totalmente } \\
\text { de acuerdo }\end{array}$ & 278 & 100 \\
\hline $\begin{array}{l}\text { Parcialmente } \\
\text { de acuerdo }\end{array}$ & 0 & 0 \\
\hline $\begin{array}{ll}\mathrm{Ni} & \text { de } \\
\text { acuerdo, } & \text { ni } \\
\text { en } & \\
\text { desacuerdo }\end{array}$ & 0 & 0 \\
\hline $\begin{array}{l}\text { Parcialmente } \\
\text { en } \\
\text { desacuerdo }\end{array}$ & 0 & 0 \\
\hline $\begin{array}{l}\text { Totalmente } \\
\text { desacuerdo }\end{array}$ & 0 & 0 \\
\hline Total & 278 & 100 \\
\hline
\end{tabular}

Fuente: Saltos (2021)

Interpretación de los resultados:

El total (100\%) de los encuestados, estuvieron totalmente de acuerdo con la inclusión de la optimización de los procesos dentro del Sistema de Gestión de Calidad, el cual conlleva a un enfoque basado en los procesos y que soluciona uno de los problemas más comunes en las organizaciones, como lo es cuando un problema o actividad no es de un único departamento, sino que intervienen varios departamentos 0 responsables diferentes.

Ítems 20. ¿Considera usted necesaria la implementación de formatos que ayuden a recabar información de todas las partes que intervienen en el laboratorio de operaciones unitarias? 
Tabla 21. Implementación de formatos que ayuden a recabar información.

\begin{tabular}{lcc}
\hline ÍTEMS & FRECUENCIA & $\begin{array}{l}\text { PORCENTAJE } \\
\%\end{array}$ \\
\hline $\begin{array}{l}\text { Totalmente de } \\
\text { acuerdo }\end{array}$ & 278 & 100 \\
$\begin{array}{l}\text { Parcialmente } \\
\text { de acuerdo }\end{array}$ & 0 & 0 \\
$\begin{array}{l}\text { Ni de acuerdo, } \\
\text { ni } \\
\text { desacuerdo }\end{array}$ & 0 & 0 \\
$\begin{array}{l}\text { Parcialmente } \\
\text { en desacuerdo }\end{array}$ & 0 & $\mathbf{0}$ \\
$\begin{array}{l}\text { Totalmente } \\
\text { desacuerdo }\end{array}$ & 0 & $\mathbf{0}$ \\
\begin{tabular}{l} 
Total \\
\hline
\end{tabular} & 278 & $\mathbf{1 0 0}$ \\
\hline
\end{tabular}

Fuente: Saltos (2021)

Interpretación. Todos los encuestados el $100 \%$ indicaron que es necesario implementar formatos que faciliten registrar toda la información relacionada al laboratorio de operaciones unitarias ya sean estos comprar a proveedores, registros de directivos y personal como también los procesos que realizan los estudiantes dentro del mismo.

\section{Discusión}

Los resultados expuestos indican que un Sistema de Gestión de la Calidad es necesario para ayudar en el funcionamiento de las actividades que se desarrollan dentro del laboratorio de operaciones unitarias de la Universidad Técnica de Manabí.

A pesar que los encuestados indican en los ítems 1,2 y que el laboratorio de operaciones Cuenta con las instalaciones y equipos necesarias para el servicio que presta y que el personal Cuenta con los equipos que se necesitan para el desarrollo de cada una de las practicas dentro de las líneas con las que Cuenta el laboratorio, se debe pensar siempre en la mejora continua y en el progreso de cada una de las instalaciones con las que Cuenta esta Universidad y el SGC es el punto de partida para esta mejora que toda organización busca para crecer, los estudiantes demuestran que tienen claro esto es así que en el ítems 4 los 278 encuestados manifiestan que es necesaria la implementación de un Sistema de Gestión de la Calidad.

Estos resultados obtenidos también muestran claramente que los estudiantes de ambas carreras que frecuentan este laboratorio tiene bien claro los elementos con los que Cuenta el laboratorio y también aquellos que hacen falta $y$ son necesarios para poder implementar el Sistema de Gestión de la Calidad 
esto se puede comprobar con los resultados del ítems 5 y 6 donde el $100 \%$ están totalmente de acuerdo.

El personal con el que Cuenta el Laboratorio de Operaciones Unitarias muestra capacidad y sapiencia dentro de sus actividades y funciones ya que poseen las habilidades y los conocimientos que se necesitan de acuerdo al SGC esto indican los estudiantes que frecuentan este laboratorio y se representa en los ítems 8 y 9. A pesar de que el personal este acto para realizar las funciones el SGA conlleva a estar en un estudio constante y esto tiene que ser asumido por la organización y por el personal.

La dirección, la gestión de los recursos y la medición de análisis y mejora del servicio deben siempre trabajar de manera eficiente $y$ permanente en cada uno de los procesos que emprenda el Laboratorio y más aún en el SGC ya que es lo que se manifiesta en el ítems 11, 12 y 13 y estos tres muestran un $100 \%$ al momento de ser preguntado a los estudiantes si estos departamentos deberían formar parte del SGC.
La comunicación tiene que ser siempre una vía que contribuya a la mejora continua dentro de cualquier organización y es por eso que al preguntarle a los estudiantes si se la tendría que tomar en Cuenta para el SGC no dudan al responder en el ítems 14 y el $100 \%$ coinciden que se tiene que incluir la comunicación entre estudiante-docente y directivos tanto a nivel de Carrera de facultad de las máximas autoridades de la Universidad.

El Espacio en donde se desarrollan las actividades de una organización tanto la infraestructura como el ambiente de trabajo ya sea este la parte tangible e intangible marcan siempre al personal que labora como al usuario del servicio es por eso que en los ítems 15 y 16 el $100 \%$ de los estudiantes indican que son dos elementos a considerarlos dentro del SGC

Los estándares de calidad, la visión integral y la optimización de los procesos juegan un papel de vital importancia ya que da personalidad y claridad a la organización a demás ayuda a reducir los gastos y genera mayor confianza y certeza en el personal y usuario es por esto que un SGC integra estos elementos en 
busca de la mejora continua y normalización de procesos, esto también lo ratifican los estudiantes al indicar el $100 \%$ en los ítems 17,18 y 19.

Cuando se habla de normalización o de un SGC no se puede dejar de lado a la información organizada y clasificada con la que debe contar una organización es por eso que en el ítems 20 se pregunta si es necesario contar con formatos que ayuden a recopilas información y mantenerla clasificada y los estudiantes coinciden al $100 \%$ que debe ser así.

\section{Conclusiones}

El Laboratorio de Operaciones Unitarias de la Universidad Técnica de Manabí cuenta con las instalaciones apropiadas para la prestación del servicio de prácticas estudiantiles pre-profesionales y profesionales, dotando para ello adecuadamente a su personal y dejando una buena imagen de la organización. Sin embargo, existe la necesidad de actualización de los equipos computarizados y la adquisición de nuevas tecnologías informáticas que permitan una mayor automatización

de

los procedimientos.

A pesar que el personal de la Universidad es competente para desempeñarse dentro de sus cargos, los retardos y restricciones de tiempo, además de los aspectos tecnológicos, privan los canales de comunicación en detrimento del desarrollo de la empatía con el cliente.

En base a lo anterior, es necesario un Sistema de Gestión de Calidad que permita la renovación de equipos, incorporación de tecnología y agilizar los procesos a fin de mejorar la calidad de los servicios, introduciendo la visión de calidad en la organización que estimule el desarrollo de una actitud de servicio por parte de los empleados. Además de disminuir los costos acarreados por la ejecución modelos de los procedimiento o repetición de los mismos por errores.

El aumento de los niveles de satisfacción fortalecerá la imagen del laboratorio, y aumentará la confianza en la relación Cliente - Proveedor, mientras que el establecimiento y cumplimiento de los estándares de calidad fortalecerán la posición del 
personal que realice prácticas en este laboratorio.

El sistema de Gestión de Calidad contempla la responsabilidad de la dirección, la gestión de los recursos, la realización del servicio, y la medición, análisis y mejora del sistema. Siendo necesario considerar la comunicación institución y usuario, infraestructura, ambiente de trabajo, estándares de calidad, visión integral y la optimización de los procesos, además de incluir el correspondiente estudio de factibilidad.

\section{Bibliografía}

Arias, F. (2017). Blog spot. Obtenido de El Proyecto de Investigación Introducción a la Metodología Científica: http://invdocumb2016.blogsp ot.com/2017/01/

Chamorro, S. (2016). Deusto Formación. Obtenido de https://www.deustoformacion. $\mathrm{com} / \mathrm{blog} /$ gestionempresas/importancia-contarcon-buenos-sistemasgestion-calidad

Flores, M. (2008). Crónicas de Mejora Contínua. Obtenido de https://www.eoi.es/blogs/mari avictoriaflores/definicion-demejora-continua/
IsoTools. (2015). ISO 9001:2008 Requisitos del Sistema de Gestión de la Calidad. Isotools. Obtenido de https://www.isotools.com.co/i so-9001-2008-requisitossistema-gestion-calidad/

López, M. (2013). Importancia de la calidad del servicio al cliente para el funcionamiento de las empresas. El Buzon Del Pacioli (82). Obtenido de https://www.itson.mx/publicac iones/pacioli/Documents/no8 2/pacioli-82.pdf

López, P. (2015). "Cómo Documentar un Sistema de Gestión de Calidad según ISO 9001:2015". Capítulo 3: "Nueva ISO 9001:2015". España: FC EDITORIAL.

Membrado. (2002). Calidad Total y Políticas de Recursos Humanos - Planteamiento teórico-metodológico.

Obtenido de https://www.tdx.cat/bitstream/ handle/10803/8807/TesisPrimeraparte.PDF?sequence $=1$ \&isAllowed $=\mathrm{y}$

Nueva ISO 9001:2015. (2018). Escuela Europea de Excelencia. Obtenido de https://www.nueva-iso-90012015.com/2018/08/como-seencuentra-formada-la-familiaiso-9000/

Surdez, E., Sandova, M. d., \& Lamoyi, C. (2018). Satisfacción estudiantil en la valoración de la calidad 
educativa universitaria.

Educación y Educadores, 21(1), 9-26

Tamayo, \& Tamayo, M. (2012). El proceso de la investigación científica. México: Limusa 